\title{
Acoustic approach of weldability for nanocomposite (nanosilica/PA6) welded by ultrasonic welding
}

\author{
Anderson Ribeiro $^{1 *}$ (D), Jaime Casanova² ${ }^{2}$ Sérgio Duarte Brandi ${ }^{3}$ and Diego de Moura Pinheiro ${ }^{3}$ \\ ${ }^{1}$ Faculdade de Tecnologia do Estado de São Paulo - FATEC, Campi Itaquera, São Paulo, SP, Brasil \\ ${ }^{2}$ Departamento de Engenharia de Materiais, Universidade Federal do Amazonas - UFAM, \\ Manaus, AM, Brasil \\ ${ }^{3}$ Departamento de Engenharia Metalúrgica e de Materiais, Universidade de São Paulo - USP, \\ São Paulo, SP, Brasil \\ *andersoncnribeiro@gmail.com
}

\begin{abstract}
Polymer matrix nanocomposites (NMP) have attracted a great interest mainly in the automotive, aerospace and medical industries since they have good mechanical properties, dimensional, thermal and chemical stability, as well as interesting electrical conductivity and cost reduction in the manufacturing process. However, welding of this class of materials presents serious challenges such as improving weldability of the joint and understanding the mechanisms responsible for coalescence. The objective of this work was to evaluate the coalescence of an NMP joint (comprising a PA6 matrix and with nanosilica of different percentages of silicon) using ultrasonic welding, as well as to perform an acoustic approach of the energy dissipation during the welding process. It is concluded that the NMP samples tend to show better coalescence as the percentage of nanosilica increases, up to a certain limit. On the other hand, the higher the content of nanoparticle the smaller the energy absorption.
\end{abstract}

Keywords: ultrasonic welding, nanocomposite, acoustics, sound spectral.

How to cite: Ribeiro, A., Casanova, J., Brandi, S. D., \& Pinheiro, D. M. (2019). Acoustic approach of weldability for nanocomposite (nanosilica/PA6) welded by ultrasonic welding. Polímeros: Ciência e Tecnologia, $29(4)$, e2019059. https://doi.org/10.1590/0104-1428.05519

\section{Introduction}

Ultrasonic welding is a widely used fusion joining process in the manufacture of polymer parts that can be used in various industrial sectors such as in the automotive, medical and aerospace industries. Studies have shown the advantages of the ultrasonic welding process in polymer-based matrix composite materials since they present better mechanical resistance and low cost when compared to other thermoplastics ${ }^{[1]}$. In ultrasonic welding, the high-frequency mechanical vibration is converted into heat by the combination of the contact surface and the intermolecular friction. In this way, the coalescence between the parts to be welded occurs ${ }^{[2]}$. Ultrasonic welding is considered as a favorable process to join thermoplastic composite materials ${ }^{[3]}$. On the other hand, polymer matrix nanocomposites have attracted a great interest mainly from industry (such as automotive, aerospace and medical industries) and researchers. The intentions are aimed at improving properties, such as mechanical, thermal and chemical properties as well as interesting electrical conductivity and cost reduction in the manufacturing process $^{[4-6]}$. Ultrasonic welding for thermoplastics is well understood, however the literature has few papers on the ultrasonic welding of nanocomposite materials ${ }^{[7,8]}$ For instance, ultrasonic welding of HDPE (Hight density polyethylene) nanocomposites with $0,3,6$ and $9 \mathrm{wt} \%$ nanoclay was investigated. It was evaluated the effect of welding parameters on the weld strength. Results showed that the maximum weld strength decreases with increasing nanoclay content ${ }^{[9]}$. Likewise, Shiu-Hung Hung evaluated some welding parameters (such as welding time, welding pressure, vibration amplitude, pressure time and joint geometry) responsible for the strength of the welded joint of a PP based composite material (glass fibre). In this research, it was observed that the composite material requires less welding energy to obtain a strong and better joint strength compared to the unfilled polymer. Also, it was shown that a higher percentage of loads contributed to intensifying the mechanical properties of the joint ${ }^{[2]}$. Similarly, Benatar and Gutowski characterized and modelled the process of ultrasonic welding for fibre reinforced composite ${ }^{[10]}$. This work aims to evaluate the coalescence of a joint of a nanocomposite material (PA6 matrix reinforced with spherical nanoparticles of silica) with different percentages of silica using ultrasonic welding, as well as to perform an acoustic energy dissipation approach.

\section{Materials and Methods}

\subsection{Materials used}

A polymer matrix composite consisting of polyamide (commercial name PA6-B260) and silica nanoparticles (with the different percentages of nanosilica, namely: 1, 5 and $7 \%$ ) 
was used. Table 1 presents the physicochemical characteristics of nanosilica, while Table 2 presents the physical properties of PA6.

To obtain the test specimens, PA6 (in pellets form) was immersed in liquid nitrogen to avoid polymer melting and to increase the hardness, and then pulverised to small grains with a mean particle size of $1.0 \mathrm{~mm}$. The powder obtained was then dried for 48 hours in an oven at $70^{\circ} \mathrm{C}$. Likewise, the nanosilica was dried under vacuum at $100^{\circ} \mathrm{C}$. Subsequently, mechanical mixing was carried out (with the addition of $1 \%$ by weight of zinc stearate as a lubricating agent) to obtain the samples with $1 \%, 5 \%$ and $7 \%$ by weight of nanosilica in PA6. After the extrusion process of the materials as mentioned above, the samples were cut into pellets. The next step was the injection of the samples into the DEMAG ${ }^{\circledR}$ Ergotech $^{\circledR}$ pro $35115^{\circledR}$ injection moulding machine, in which 50 test bodies were injected for each percentage of nanosilica. Table 3 shows the mechanical properties for each sample group of the experiment.

\subsection{Welding parameters}

The welding parameters used in the experiment were determined after a welding process involving 30 specimens (CPs) for each condition, for a total of $120 \mathrm{CPs}$. The defects in the welds were evaluated, and it was possible to construct the operating window for the process and to determine the appropriate parameters. Thus, a welding pressure of $15 \mathrm{psi}$, with varying welding time, from 0.2 to 0.6 seconds and a 0.1 -second interval was employed for all the samples. Before the welding process, all the materials were stored in an oven at $60{ }^{\circ} \mathrm{C}$ for at least 48 hours. Figure 1 shows how the test bodies were attached to the welding equipment. The measurement of the welded area was done using Image J software.

\subsection{Tests}

The assay to evaluate shear strength was performed according to ASTM D 638 standard. 15 samples for each percentage of nanosilica were evaluated in the shear

Table 1. Physico-chemical characteristics of nano-silica.

\begin{tabular}{lcc}
\hline \multicolumn{1}{c}{ Properties } & Unit & Value \\
\hline Specific surface area (BET) & $\mathrm{m}^{2} / \mathrm{g}$ & $200 \pm 25$ \\
Average particle size & $\mathrm{Nm}$ & 12 \\
Compressed density (DIN EN ISO 787/11) & $\mathrm{g} / \mathrm{l}$ & close to 50 \\
Humidity & $\%$ in weight & $\leq 1.5$ \\
Loss in fire $2 \mathrm{~h}$ a $1000^{\circ} \mathrm{C}$, Based on a dried material by $2 \mathrm{~h} \mathrm{a} 105^{\circ} \mathrm{C}$ & $\%$ in weight & $\leq 1.0$ \\
$\mathrm{pH}$ dispersion of $4 \%$ & \% in weight & 3.74 .7 \\
$\mathrm{SiO}_{2}$ level & & $\geq 99.8$ \\
\hline
\end{tabular}

Table 2. Mechanical Properties of Polyamide (PA6).

\begin{tabular}{lccc}
\hline \multicolumn{1}{c}{ Properties } & B 260 & Unit & ASTM Standard \\
\hline Tensile stregth & s 75 & MPa & D638 \\
Tensile modulus & c 50 & MPa & D638 \\
Elongation & s 2900 & & D 638 \\
Flexural strength & c 1300 & $\%$ & D790 \\
Flexural modulus & s 50 & & MPa \\
c $>120$ & & $\mathrm{D} 790$ \\
Rockwell hardness & s 100 & $\mathrm{MPa}$ & $\mathrm{D} 785$ \\
Impact resistance Izod & $\mathrm{c} 40$ & $\mathrm{R}$ & $\mathrm{D} 256$ \\
\end{tabular}

Table 3. Mechanical properties of the nanocomposite.

\begin{tabular}{|c|c|c|c|c|c|}
\hline $\begin{array}{c}\text { Percentage of } \\
\text { nanosilica }\end{array}$ & Maximum load (N) & UTS (N/mm²) & YS $\left(\mathbf{N} / \mathbf{m m}^{2}\right)$ & Along. (\%) & $\mathbf{E}\left(\mathbf{N} / \mathbf{m m}^{2}\right)$ \\
\hline $0 \%$ & 2599.15 & 59.21 & 29.44 & 424.62 & 189.07 \\
\hline $1 \%$ & 2604.65 & 59.35 & 22.38 & 72.12 & 573.53 \\
\hline $5 \%$ & 2068.42 & 47.15 & 20.69 & 84.30 & 255.21 \\
\hline $7 \%$ & 2565.42 & 58.45 & 23.75 & 36.44 & 629.78 \\
\hline
\end{tabular}

UTS = Ultimate tensile strength; YS = Yield strength; $\mathrm{E}=$ modulus of elasticity. 
strength test. The speed and load for performing the test were $5 \mathrm{~mm} / \mathrm{min}$ and $1.47 \mathrm{~N}$, respectively.

\subsection{Acoustic analysis}

Three recordings were made per sample group including three recordings with the sonotrode without pressing the sample. The recordings of the subharmonics were performed using a dynamic unidirectional (cardioid)

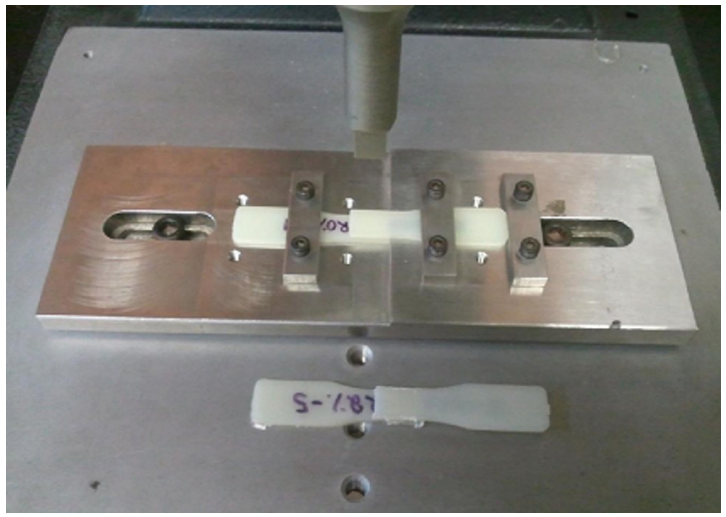

Figure 1. Fixation of test bodies.

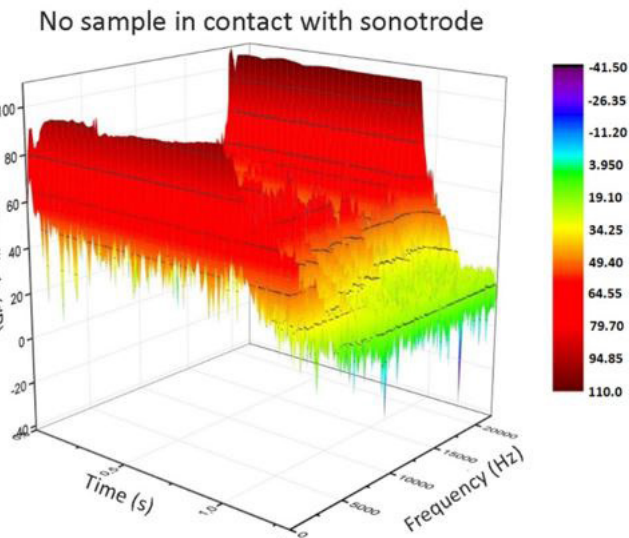

(a)

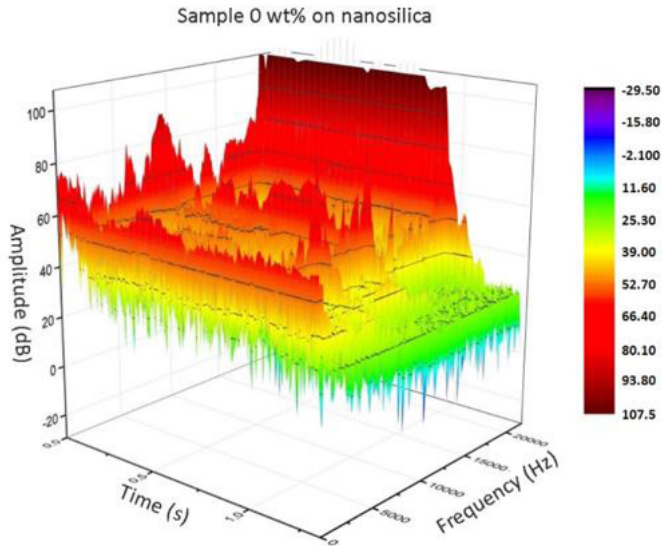

(b) microphone, a rigid microphone stand, an analogue-digital converter and recording software. The microphone was positioned with an inclination of $45^{\circ}, 10 \mathrm{~cm}$ from the point of interest (sonotrode and sample). The positioning was done empirically. Spectral results of the recording were analyzed in real time and the info were processed by commercial software named SpectraLAB: FFT - Spectral Analysis System ${ }^{[11]}$ which is able to process data by fast Fourier transform analysis (FFT).

\section{Results and Discussions}

The spectra corresponding to the recordings of the sound emitted for each welding condition can be observed in Figure 2. In this figure, the spectra were plotted using "amplitude intensity versus frequency" since this provides conditions to identify the time for a specific frequency in addition to estimating the energy of the signal in the spectrum.

\subsection{Acoustic approach of the welding energy}

The quality of the welded joint of the polymer matrix composite material (CMP) is related to several factors such as the heat generated by the action of the tool, the incorporation of the load/polymer and the mechanical properties of the CMP, besides the melt area during the

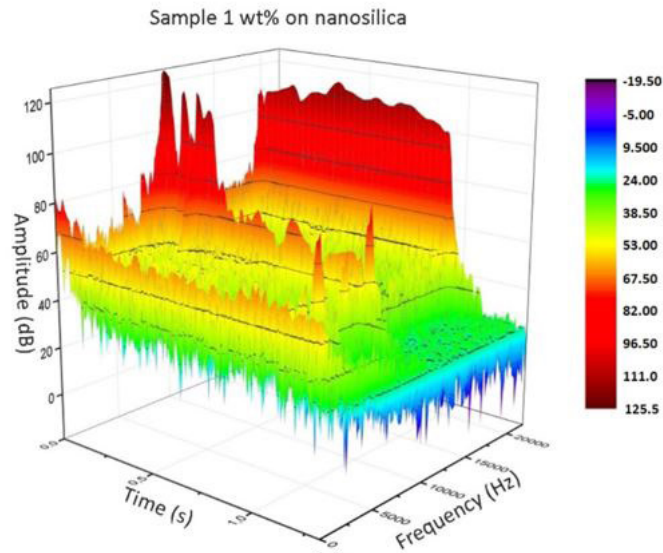

(c)

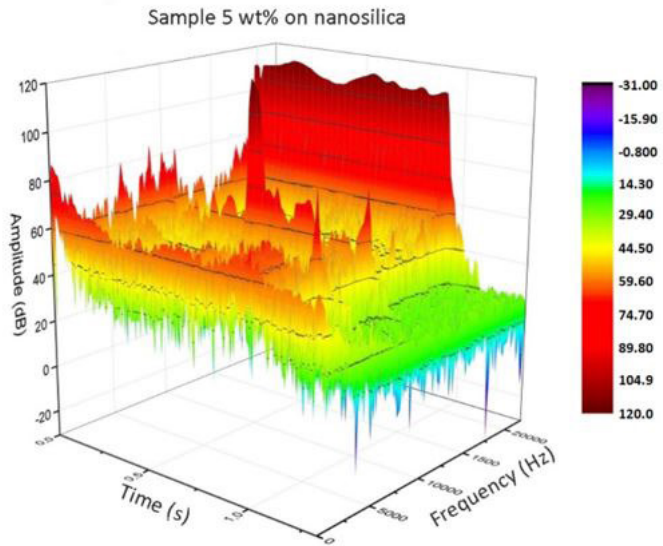

(d)

Figure 2. Sound spectral emitted in each welding condition. 
welding process. Thus, ultrasonic welding is the result of several regimes in which acoustic contact drop, reduction in static pressure, and changes in amplitude and vibration frequency occur ${ }^{[12,13]}$.

In order to evaluate the spectra corresponding to the ultrasonic oscillation generated by the sonotrode without sample (SA), with pure sample (AP), and other welding conditions, samples with $1 \%$ (A1), 5\% (A5) and 7\% (A7) nanosilica, the model that treats the level of intensity of spectrum (ISL - Intensity Spectrum Level), were used for several frequency bands. Thus, ISL is the sound wave intensity for $1 \mathrm{~Hz}$ frequency band and can be determined by Equation 1.

$$
I S L=10 \log \frac{I(\text { in } 1 \mathrm{~Hz} \text { band }) 1 \mathrm{~Hz}}{I_{\text {ref }}}
$$

On the other hand, the conditions analyzed in this present work resulted in spectra with several band levels (BL); this implies that the intensity of a BL is determined by Equation 2 .

$$
B L=10 \log \frac{I(\text { band de } 1 \mathrm{~Hz}) 1 \mathrm{~Hz}}{I_{\text {ref }}}+10 \log \frac{\Delta f}{1 \mathrm{~Hz}}
$$

Otherwise, BL may be related to ISL as presented in Equation 3.

$$
B L=I S L_{a v e}+10 \log (\Delta f)
$$

where: $\Delta f=f_{2}-f_{1}$, of $I S L_{\text {ave }}$ corresponds to the average ISL.

Finally, the integration of Equation 3 corresponds to the area of the spectra shown in Figure 2 (considering the limits of integration from $800 \mathrm{~Hz}$ to $20 \mathrm{kHz}$ ) in which it is possible to compare the eminent energy of the sound wave for each studied welding condition. The calculated energy is shown in Figure 3.

From Figure 3 it can be seen that the energy intensity emitted in sound waveform during AP welding is $7 \%$ lower than the energy estimated for AS. In other words, the AP welding condition absorbed approximately $93 \%$ of

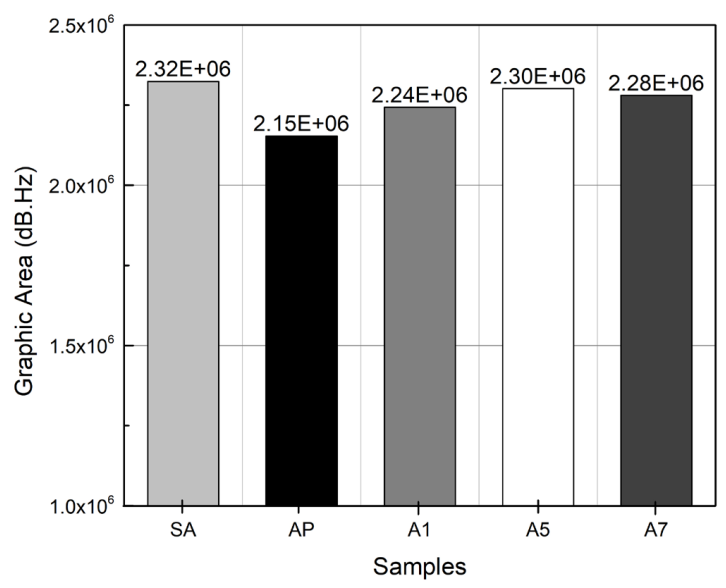

Figure 3. Estimated energy in the sound emissions in each welding condition. the vibration energy emitted by the ultrasonic oscillation generated by the sonotrode (OUS). To explain the factors that contributed to this great disparity, the dissipation and absorption of energy will be evaluated. Considering the model presented in Figure 4, we see that the ultrasonic vibration is transmitted to the material by the sonotrode and induces the interface where the joint will occur (region 6) ${ }^{[14]}$. In the same way, the welding is influenced by the acoustic impedance of both the nanocomposite and the OUS. The intensity of the ultrasonic oscillation generated by the OUS is given by:

$$
I_{0}=2 \pi^{2} f^{2} A_{0}^{2} \rho_{0} c_{0}
$$

In the SA case, the absorption of $\mathrm{I}_{0}$ occurred only at the base (region 4 of Figure 4), disregarding the losses by the environment, so all the vibration dissipated by the system in the form of sound energy was captured and converted into ISL by the recording software. On the other hand, for AP welding, $\mathrm{I}_{0}$ suffered losses due to the acoustic impedance of the polymer $\left(Z_{1}=\rho_{1} c_{1}\right)$, this resulted in $\mathrm{I}_{1}$ (intensity of the oscillation at interface 6) that was responsible for the vibration at the polymer/polymer interface. Thus, the increase in temperature occurred due to the absorption of the mechanical vibrations, a reflection of the vibration in the bound region and the friction at the surface. The combination of absorption and vibration reflections corresponds to the acoustic attenuation of the material. The calculation of the absorption coefficient of the wave can be done by using Equation 5.

$$
A(f)=-20 * \log \left(\frac{V_{2}}{V_{1}}\right)
$$

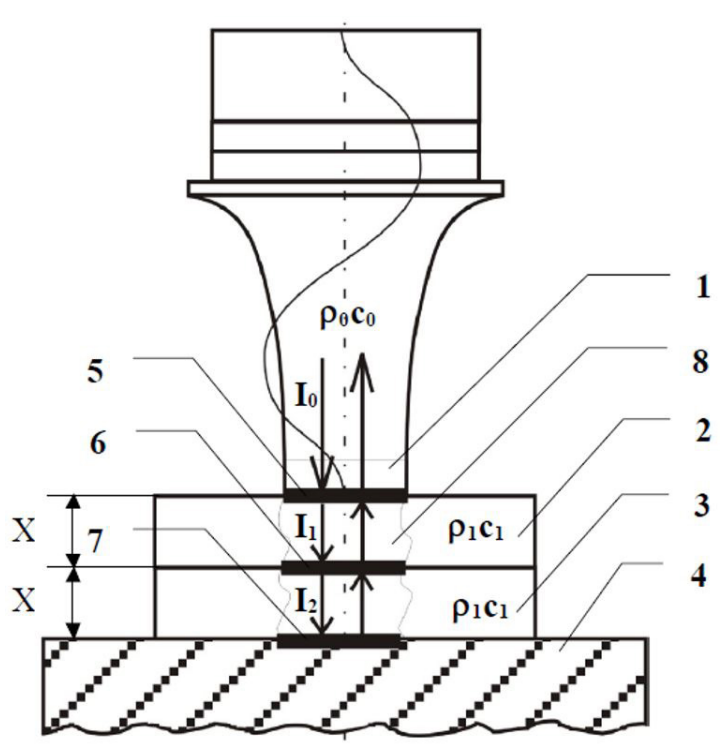

Figure 4. Schematic representation of vibration through sonotrode, workpiece and anvil ${ }^{[14]}$. (1) working welding tool (sonotrodo); (2) and (3) acoustical impedance of the materialsZ $\left(Z_{1}=\rho_{1} c_{1}\right)$; $Z_{0}=\rho_{0} c_{0}$-acoustical impedance of the ultrasonic oscillatory system; (4) support; (5) welded materials; (6) material/material interface; (7) reflection of a supersonic wave from an interface of medium; (8) energy absorption zone; $\mathrm{X}=$ thickness. 
Knowing that:

$$
\frac{V_{2}}{V_{1}}=\alpha z
$$

where $A(f)$ is the acoustic absorption, given in $\mathrm{dB} . \alpha$ is the absorption coefficient in $\mathrm{dB} / \mathrm{mm}$, and $\mathrm{z}$ is the distance travelled by the wave, in $\mathrm{mm}$.

The relation $\frac{V_{2}}{V_{1}}$ can be considered as the acoustic attenuation. Table 4 presents the results obtained in the determination of the acoustic attenuation and the absorption coefficient using transducers of 5 and $10 \mathrm{MHz}$.

Research has shown that acoustic attenuation in PMCs depends on the size of the charge of the particles, the degree of crystallinity (increasing the degree of crystallinity tends to reduce acoustic dissipation) and the incorporation of the charge in the matrix, i.e. the interaction between polymer/load ${ }^{[15]}$. It is summarized that CMPs with small particle sizes present better acoustic attenuation and higher volumetric fraction conditions for the same particle size, which results in higher wave attenuation compared to CMP with larger particles. This is because the increase in the number of particles in the matrix has a larger surface area for wave propagation ${ }^{[16]}$. In Table 4 we observe that the values of the absorption coefficients $(\alpha)$ and the acoustic attenuation $\frac{V_{2}}{V_{1}}$ for each studied condition are very close, however, sample A5 presents the smallest value of $\alpha$ and higher value of $\frac{V_{2}}{V_{1}}$. These data justify the higher energy value dissipated for A5 as seen in Figure 3. On the other hand, it can be seen (in Table 4) that sample A1 has similar $\alpha$ value with sample A7, but higher than sample A5. Some of the factors that contributed to the behaviour of $\alpha$ in A1 were the homogeneous distribution and the better incorporation of the nanoparticles in the CMP, this also explains the lower energy dissipated value for A1 (see Figure 3). However, for A7, a lower value of $\alpha$ was expected, since it had a higher fraction of nanosilica, but there was the formation of agglomerates and the inhomogeneous distribution of the particles.

\subsection{Weld area and shear strength}

It is possible to observe in Figure 5 that samples with 5\% and $7 \%$ nanosilica presented higher welded area compared to the other welding conditions for all the welding times analyzed.

Analyzing Figure 5, it is possible to highlight the influence of the percentage of nanosilica on the welded area. In this way, it is observed that the shear strength increases with increasing load percentage. Figure 6 and 7 depict the shearing surface from each welded condition. It can be observed the size of the molten pool and their distribution in the weld. In Figure 6 the melted region grows up from the edge to the center, but not completely, while in Figure $6 \mathrm{~b}$

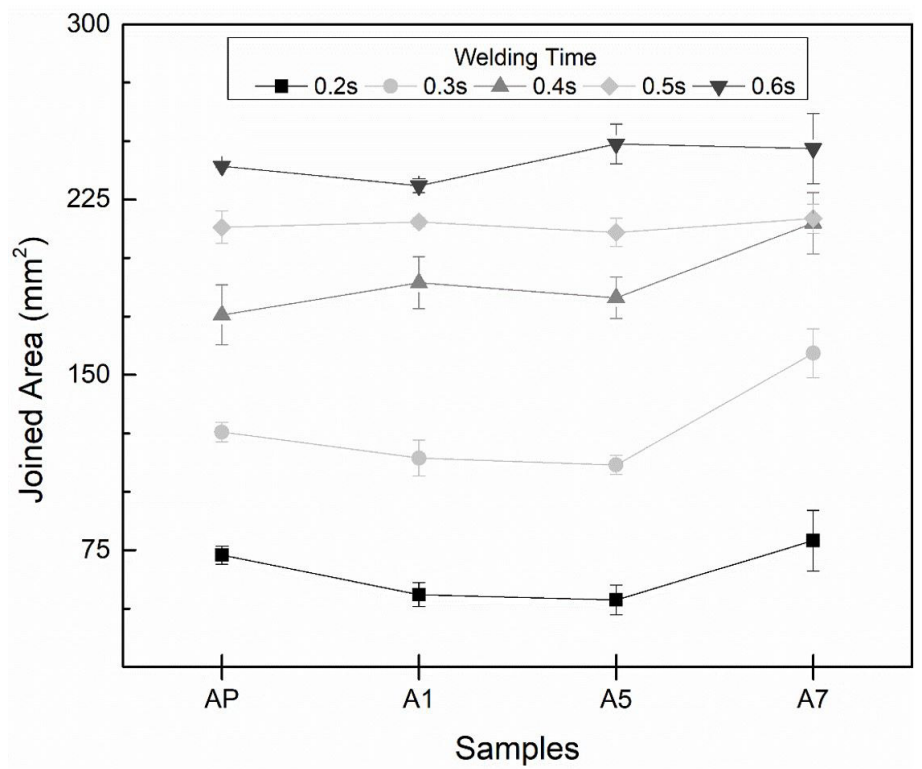

Figure 5. Weld area analysis for each welding condition.

Table 4. Evaluation of sound attenuation.

\begin{tabular}{|c|c|c|c|c|c|c|c|c|}
\hline \multicolumn{4}{|c|}{ Transducer of $5 \mathrm{MHz}$} & \multicolumn{5}{|c|}{ Transducer of $10 \mathrm{MHz}$} \\
\hline Sample & $\mathbf{V}_{1}(\mathbf{V})$ & $\mathbf{V}_{2}(\mathrm{~V})$ & $\frac{V_{2}}{V_{1}}$ & $\alpha(\mathrm{dB} / \mathrm{mm})$ & $V_{1}(V)$ & $\mathbf{V}_{2}(\mathbf{V})$ & $\frac{V_{2}}{V_{1}}$ & $\alpha(\mathrm{dB} / \mathrm{mm})$ \\
\hline $\mathbf{A P}$ & 1.80 & 0.262 & 0.15 & 0.0221 & 0.65 & 0.075 & 0.12 & 0.0175 \\
\hline A1 & 2.10 & 0.275 & 0.13 & 0.0198 & 1.24 & 0.181 & 0.15 & 0.0221 \\
\hline A5 & 1.86 & 0.212 & 0.11 & 0.0173 & 0.825 & 0.093 & 0.11 & 0.0171 \\
\hline A7 & 2.23 & 0.325 & 0.15 & 0.0221 & 1.24 & 0.168 & 0.14 & 0.0205 \\
\hline
\end{tabular}




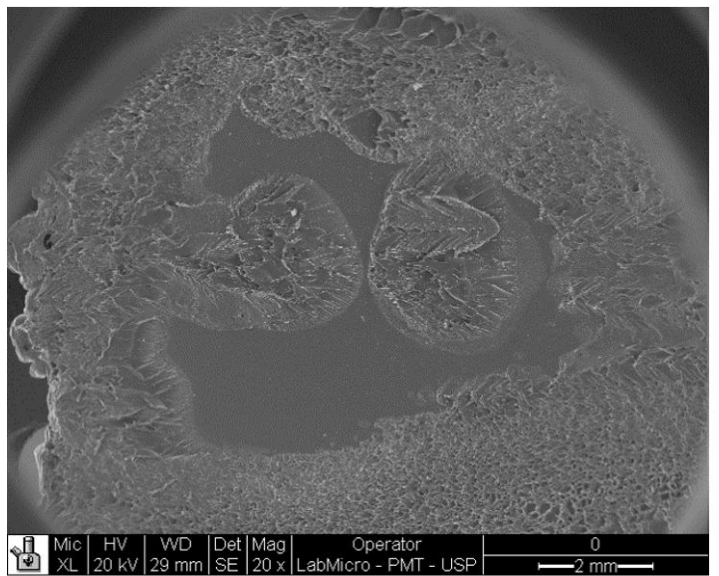

(a)

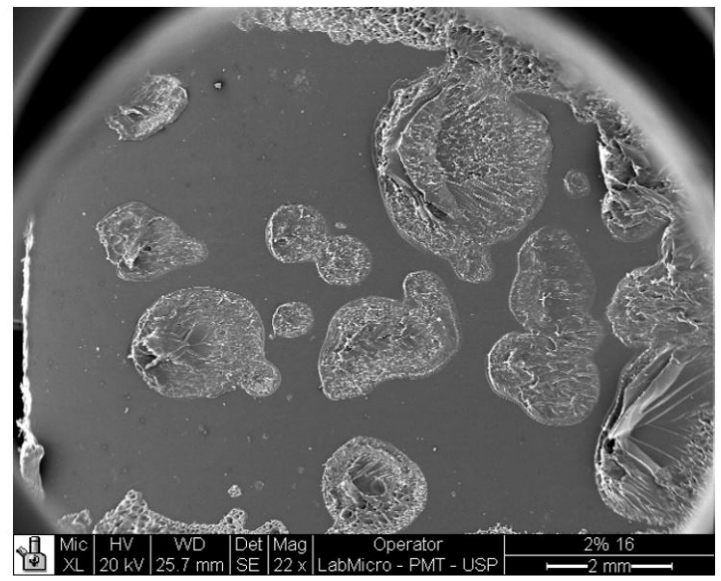

(b)

Figure 6. (a) SEM image of sheared surface for sample with $0 \%$ wt. nanosilica; (b) SEM image of sheared surface for sample with $1 \%$ wt. nanosilica.

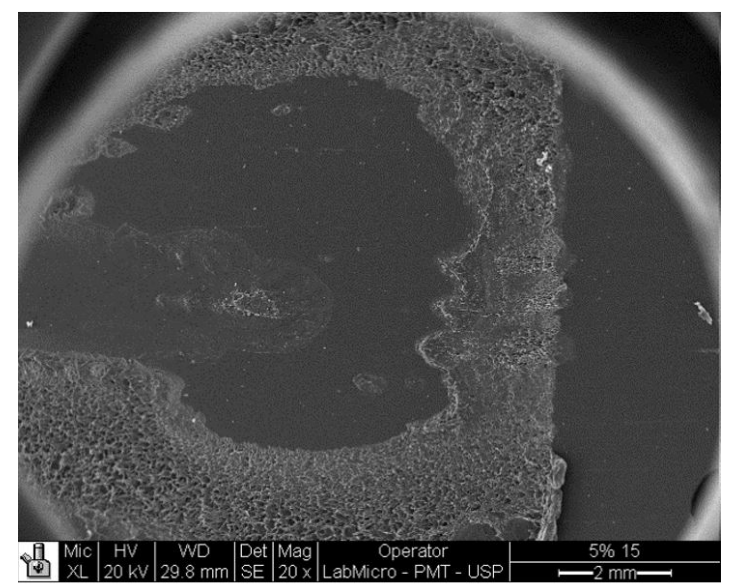

(a)

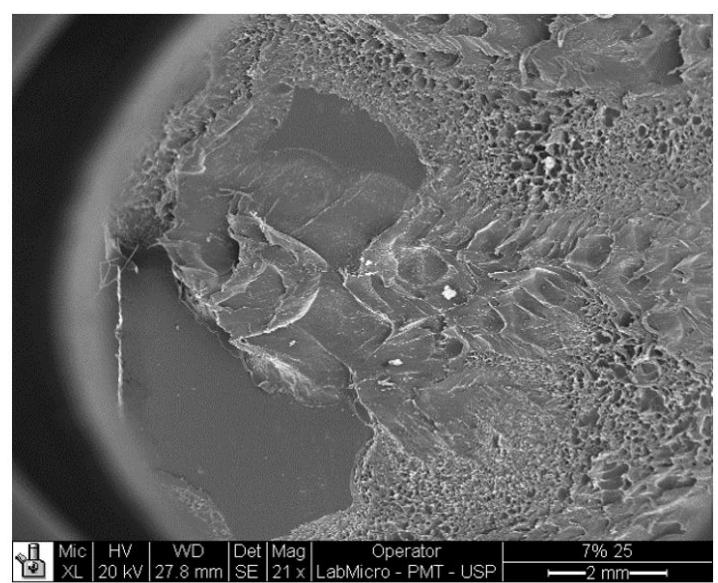

(b)

Figure 7. (a) SEM image of sheared surface for sample with 5\% wt. nanosilica; (b) SEM image of sheared surface for sample with $7 \%$ wt. nanosilica.

we can see small melted regions distributed inside the weld. However, the molten area tends to increase for samples with higher percentage of nanosilica (Figure 7). It is commonly accepted that the addition of nano-fillers into semi-crystalline polymers can accelerate their crystallization behavior and increase the thermal conductivity of polymer matrix ${ }^{[17-19]}$.

Comparing the results presented in Figure 3 with those of Figure 5, we can see that the welded conditions with the highest emission energy ( $\mathrm{A} 5$ and $\mathrm{A} 7$ ) were the ones with the best mechanical weldability (second percentage of the welded area - Figure 5). This is because the charge has a dissipative modulus higher than that of the polymer ${ }^{[20]}$. Thus, the energy was dissipated by vibration at the welded interface. Finally, the nanocomposite with higher load percentage requires less amount of vibrational energy to be welded (than the pure polymer), since when the load vibrates in phase with the sonotrode, more energy is transferred to the matrix ${ }^{[21]}$. On the other hand, the pure polymer is a more viscous material, with a high dissipative modulus (E"), and does not vibrate in phase with the sonotrode. Consequently, the energy rate transferred to the joint to be welded is lower ${ }^{[22]}$.

\section{Conclusions}

When analyzing the results obtained in the acoustic energy dissipation approach associated with the shear strength test, it was possible to conclude that:

- Pure samples (without the addition of nanosilica) welded by ultrasound absorbed about $90 \%$ of the vibration energy emitted by ultrasound oscillation by the sonotrode. This was due to the difference in acoustic impedance between the polymer, the sonotrode and the fixed sample base. Therefore, the main factors responsible for welding were the absorption of mechanical vibrations and friction at the surface interface;

- The sample with 5\% nanosilica (A5) presented low value of absorption coefficient and high value of acoustic attenuation due to the volumetric fraction of nanosilica, which causes a larger surface area for sound wave scattering. The sample with 7\% nanosilica (A7) did not show the same behaviour as A5 because there was the formation of agglomerates and the distribution of inhomogeneous particles; 
- Samples A5 and A7 showed better weldability, although they absorbed less amount of vibrational energy;

- The dissipative module of the nanosilica is superior to the modulus of the polymer, i.e. when the charge vibrates in phase with the oscillation transmitted by the sonotrode, a greater amount of energy is transmitted to the interface that will be welded.

\section{References}

1. Rashli, R., Bakar, E. A., Kamaruddin, S., \& Othman, A. R. (2013). A review of ultrasonic welding of thermoplastic composites. Caspian Journal of Applied Sciences Research, 2(3), 1-16. Retrieved in 2019, July 11, from http://www.cjasr. com/images/manuscripts/2013/03/cjasr/01_CJASR-12-16-307. pdf

2. Liu, S. J., Chang, I.-T., \& Hung, S.-W. (2001). Factors affecting the joint strength of ultrasonically welded polypropylene composites. Polymer Composites, 22(1), 132-141. http://dx.doi. org/10.1002/pc. 10525 .

3. Villegas, I. F., \& Palardy, G. (2017). Ultrasonic welding of CF/ PPS composites with integrated triangular energy directors: melting, flow and weld strength development. Composite Interfaces, 24(5), 515-528. http://dx.doi.org/10.1080/09276 440.2017.1236626.

4. Lehmann, B., Schlarb, A. K., Friedrich, K., Zhang, M. Q., \& Rong, M. Z. (2008). Modelling of mechanical properties of nanoparticle-filled polyethylene. International Journal of Polymeric Materials and Polymeric Biomaterials, 57(1), 81100. http://dx.doi.org/10.1080/00914030701337232.

5. Han, Z., \& Fina, A. (2011). Thermal conductivity of carbon nanotubes and their polymer nanocomposites: a review. Progress in Polymer Science, 36(7), 914-944. http://dx.doi. org/10.1016/j.progpolymsci.2010.11.004.

6. Kim, H., Miura, Y., \& Macosko, C. W. (2010). Graphene/ polyurethane nanocomposites for improved gas barrier and electrical conductivity. Chemistry of Materials, 22(11), 34413450. http://dx.doi.org/10.1021/cm100477v.

7. Arul Selvan, S. G., Rajasekar, R., Kalidass, M., \& Selwin, M. (2017). Vibration and ultrasonic welding behaviour of polymers and polymer composites: a review. Journal of Chemical and Pharmaceutical Sciences, 2017(3), 55-61. Retrieved in 2019, July 11, from https://www.jchps.com/specialissues/2017\%20 Special\%20Issue\%203/MKCE_MECH\%2012.pdf

8. Lin, L., \& Schlarb, A. K. (2015). Vibration welding of polypropylene-based nanocomposites - The crucial stage for the weld quality. Composites Part B, Engineering, 68, 193-199. http://dx.doi.org/10.1016/j.compositesb.2014.08.052.

9. Flowers, S., Thomas, J., Mokhtarzadeh, A., \& Benatar, A. (2006). Study of ultrasonic welding of hdpe-based nanoclay composites. In ANTEC 2006 Plastics: Annual Technical Conference Proceedings (pp. 2189-2193). Charlotte: Society of Plastics Engineers.
10. Benatar, A., \& Gutowski, T. G. (1989). Ultrasonic welding of PEEK graphite APC-2 composites. Polymer Engineering and Science, 29(23), 1705-1721. http://dx.doi.org/10.1002/ pen.760292313.

11. SpectraLAB. (1998). FFT Spectral Analysis System. Version 4.32.14. Sound Technology.

12. Lyashko, F. E., \& Sokolova, O. F. Method of ultrasound welding thermoplastic. Patent 2220917 kl. B 29 C 65/08. Russia.

13. Raza, S. F. (2015). Ultrasonic welding of thermoplastics (Doctoral thesis). The University of Sheffield, United Kingdom.

14. Khmelev, V. N., Slivin, A. N., \& Abramov, A. D. (2017). Model of process and calculation of energy for a heat generation of a welded joint at ultrasonic welding polymeric thermoplastic materials. In 8th Siberian Russian Workshop and Tutorial on Electron Devices and Materials (pp. 316-322). Erlagol: IEEE. http://dx.doi.org/10.1109/SIBEDM.2007.4292995

15. Grewe, M. G., Gururaja, T. R., Shrout, T. R., \& Newnham, R. E. (1990). Acoustic properties of particle/polymer composites for ultrasonic transducer backing applications. IEEE Transactions on Ultrasonics, Ferroelectrics, and Frequency Control, 37(6), 506-514. http://dx.doi.org/10.1109/58.63106. PMid:18285071.

16. Nargund, S. (2016). Evaluation of stress wave attenuation in a polymer matrix composite using finite element analysis technique. In ASME 2016 International Mechanical Engineering Congress and Exposition (pp. 1-9, Paper No: IMECE2016-67055, V010T13A018). Phoenix: ASME. http://dx.doi.org/10.1115/ IMECE2016-67055

17. Garcia, M., Vliet, G. V., Jain, S., Zyl, W. E. V., \& Boukamp, B. (2004). Polypropylene/SiO2 nanocomposites with improved mechanical properties. Reviews on Advanced Materials Science, 6(2), 169-175. Retrieved in 2019, July 11, from https://research. tue.nl/en/publications/polypropylenesio2-nanocompositeswith-improved-mechanical-propert

18. Zhou, T. H., Ruan, W. H., Mai, Y. L., Rong, M. Z., \& Zhang, M. Q. (2008). Performance improvement of nano-silicapolypropylene composites through in-situ cross-linking approach. Composites Science and Technology, 68(14), 28582863. http://dx.doi.org/10.1016/j.compscitech.2007.10.002.

19. Pflug, G., Gladitz, M., \& Reinemann, S. (2009). Wärme besser leiten. Kunststoffe, 12, 54-60. Retrieved in 2019, July 11, from https://www.kunststoffe.de/_storage/asset/538981/ storage/master/file/5806046/download/W\%C3\%A4rme\%20 besser\%20leiten.pdf

20. Rosato, D. V. (1990). Plastics processing data handbook. New York: Van Nostrand Reinhold. http://dx.doi.org/10.1007/97894-010-9658-4.

21. Fitzgibbon, W. E., \& Wheeler, M. F. (1992). Wave propagation and inversion. Philadelphia: SIAM.

22. Benatar, A., \& Cheng, Z. (1989). Ultrasonic welding of thermoplastics in the far-field. Polymer Engineering and Science, 29(23), 1699-1704. http://dx.doi.org/10.1002/pen.760292312.

Received: July 11, 2019

Revised: Jan. 16, 2020

Accepted: Feb. 19, 2020 